\title{
Effect of Pelvic Floor Muscle Rehabilitation in Preventing Recurrent Vulvovaginal Candidiasis and Recurrent Bacterial Vaginosis
}

\author{
Cheng Fang ${ }^{1}$, Yang Yun-Jie ${ }^{1}$, Shi Wen-jing ${ }^{1}$, Wang Xue-Ying ${ }^{2}$, Ma Le ${ }^{2, *}$ \\ ${ }^{1}$ Maternity and Child Care Hospital of Huaian in Jiangsu Province, Huaian, China \\ ${ }^{2}$ Beijing Obstetrics and Gynecology Hospital, Capital Medical University, Beijing, China
}

Email address:

Malef163bj@qq.com (Ma Le)

${ }^{*}$ Corresponding author

\section{To cite this article:}

Cheng Fang, Yang Yun-Jie, Shi Wen-jing, Wang Xue-Ying, Ma Le. Effect of Pelvic Floor Muscle Rehabilitation in Preventing Recurrent Vulvovaginal Candidiasis and Recurrent Bacterial Vaginosis. Journal of Gynecology and Obstetrics. Vol. 6, No. 4, 2018 , pp. $94-97$. doi: $10.11648 /$ j.jgo.20180604.14

Received: July 10, 2018; Accepted: July 24, 2018; Published: September 13, 2018

\begin{abstract}
Objective To investigate the effect of pelvic floor muscle rehabilitation on recurrent vulvovaginal candidiasis (RVVC) and recurrent bacterial vaginosis (RBV). Methods Between January 2014 and July 2015, 121 cases of RVVC and RBV were selected, and the patients were randomly divided into control group and experimental group. For the control group of RVVC: miconazole suppository and vaginal delivery were utilized in every patient, 1 piece per night, the period of treatment was 7 to 14 days; oral intake of fluconazole was used again after 72 hours. Clotrimazole suppository or fluconazole was still chosen for the maintenance treatment: (1) 1 piece per week and lasted 6 months for every case; (2) $400 \mathrm{mg}$ of itraconazole was used, 1 time every month or $100 \mathrm{mg}$ was used once a week, the period of treatment lasted for 6 months. RBV: oralintake of metronidazole or clindamycin was chosen. One piece of metronidazole suppository by vaginal utilization was still needed per night, or $2 \%$ clindamycin ointment was used by inunction for 7 days. Experimental group: pelvic floor muscle rehabilitation. Results Before rehabilitation treatment, there were 4 cases with level 4 or 5 of type I muscle strength and 8 cases level 4 or 5 of type II muscle strength. After the treatment, there were 59 cases with level 4 or 5 of type I muscle, and 60 cases with level 4 or 5 of type II muscle $(\mathrm{P}<0.001)$. There were no statistical differences on the treatment of anterior vaginal wall prolapse. Before the treatment, the number of anterior vaginal wall prolapse was 53 cases, the number was 49 cases after treatment $(\mathrm{P}=0.328)$. Before treatment, the number of posterior vaginal wall prolapse was 25 cases, the number was 11 cases after treatment $(\mathrm{P}=0.005)$. The number of Uterine prolapse was 14 cases, the number was 2 cases after treatment $(\mathrm{P}=0.002)$. Compared with the group with drug treatment, the rate of relapse in vaginitis was decreased significantly after pelvic floor rehabilitation within 1 year, 61 patients with vaginitis, there were only 2 cases of recurrences were observed after treatment $(\mathrm{P}<0.05)$. Conclusion Pelvic floor rehabilitation treatment could improve the pelvic floor function significantly, and also get significant recovery of sagging and prolapse in vagina which was injured by pregnancy and childbirth. Compared with the drug treatment group, the rate of relapse in RVVC and RBV was decreased significantly after pelvic floor muscle rehabilitation.
\end{abstract}

Keywords: Pelvic Organ Prolapse, Pelvic Floor Muscle Rehabilitation, Recurrent Vulvovaginal Candidiasis, Recurrent Bacterial Vaginosis

\section{Introduction}

Vulvovaginal candidiasis (VVC) is an inflammation of the vulvovaginal skin caused by Candidiasis, if symptoms appear repeatedly within one year and mycological evidence confirms that VVC has occurred four times or more, this is called recurrent vulvovaginal candidiasis. Yeast Disease (RVVC) [1-4]. The treatment of VVC is mainly based on drugs. Because there are many transverse folds in the vaginal mucosa, it is easy to hide the bacteria. If the dosage is not enough or does not meet the treatment requirements, it will not be easy to completely kill the sterilization wire and spores, and 
some patients are prone to drug resistance. $5 \% \sim 10 \%$ of VVC patients are converted to RVVC [1]. Bacterial vaginosis (BV) is a clinical syndrome characterized by an increase in Gram-negative anaerobic bacteria and a reduction and/or a disappearance of Lactobacillus vaginalis, and is a mixed infection caused by dysregulation of the normal flora in the vagina. Recurrent $\mathrm{BV}$ is called recurrent bacterial vaginosis (RBV) [1]. Select RVVC and RBV cases in the non-acute phase of inflammation, use the pelvic floor rehabilitation techniques to give rehabilitation treatment, and compare the inflammatory recurrence before and after pelvic floor rehabilitation.

\section{Documents and Methods}

\subsection{Subjects}

Randomly selected RVVC and RBV patients from January 2014 to July 2015 who were not satisfied with the effect of drug treatment in Huai'an Maternal and Child Health Hospital, and who were voluntarily received pelvic floor rehabilitation therapy. A total of 61 patients entered the observation group and were all married, procreated with an average of 28.3 years old. There were 32 cases of vaginal delivery and 29 cases of cesarean section. The average weight gain during pregnancy was $15.5 \mathrm{~kg}$, and the average weight of newborns was $3445 \mathrm{~g}$. There were 42 patients with RVVC, 15 patients with RBV, and 4 patients with alternating RVVC and RBV or almost simultaneous morbidity. The control group was a drug treatment group. 60 patients with RVVC and RBV diagnosed in our hospital at the same time were randomly selected and voluntarily prescribed drug treatment. There were 38 patients with RVVC, 16 patients with RBV, and 6 patients with RVVC.

Alternating or nearly simultaneous RBV morbidity, with an average of 27.4 years, including 39 cases of vaginal delivery and 21 cases of cesarean section; The average weight gain during pregnancy was $14.6 \mathrm{~kg}$, and the average weight of newborns was $3260 \mathrm{~g}$.

The observation group (pelvic floor rehabilitation) was enrolled in the following conditions: (1) Clinical and laboratory bacteriological examination confirmed VVC and BV. (2) After treatment with antifungal and anti-gram-negative anaerobic bacteria, the effect is poor and the annual incidence is $\geq 4$ times. (3) exclude diabetes and immune dysfunction. (4) Non-electrical stimulation contraindications (including acute vaginal inflammation, pregnancy, menstrual period, abnormal vaginal bleeding, installation of pacemakers, urogenital malignancies, epilepsy and dementia, etc.). (5) Not acute vaginal inflammation.

Control group (drug treatment) was enrolled in the following conditions: (1) clinical and laboratory bacteriological examination confirmed as VVC and BV, annual incidence of $\geq 4$ times. (2) Patients voluntarily choose drug treatment. (3) Exclude pregnancy, abnormal vaginal bleeding, genitourinary malignancy, etc.

Patients were voluntarily and fully informed, and the subjects all signed informed consent. This research passed the ethics committee of Huai'an Maternal and Child Health Hospital. It will be agreed that the follow-up period after treatment is 1 year, and the study will be completed by July 2016.

\subsection{Diagnostic Criteria}

\subsection{1. $R V V C$}

Symptoms and bacteriologically confirmed VVC recidivate 4 times and more [1].

\subsubsection{BVUsing Amesel Diagnostic Criteria}

If three of the following four items are positive can be diagnosed as BV: (1) Uniform, thin, white vaginal secretions, adherent to the vaginal wall. (2) The clue cells are positive. (3) The vaginal secretions have a $\mathrm{pH}>4.5$. (4) Positive amine odor test. Patients with recidivation $\geq 4$ times were enrolled as RBV cases.

\subsection{Evaluation of Efficacy}

Pelvic floor rehabilitation and drug treatment were followed up for 1 year and 1 time/month telephone follow-up. In case of vaginal discharge abnormalities, vaginal itching, and odor, the vaginal secretions for bacteriological examination are given; if the enrolled personnel consciously have the above symptoms, they may seek treatment on their own, and if there is no symptoms, they will not receive medical intervention. Total effective rate $=$ (number of cured people + effective number) / total number of treatments $\times$ $100 \%$.

\subsubsection{RVVC Efficacy Evaluation}

Cure: The clinical symptoms completely disappeared and the Candida microscopic examination was negative. Effective: Significantly reduced clinical symptoms and negative Candidiasis microscopic examination. Invalid: no significant changes in clinical symptoms or aggravate, microscopic examination of Candidiasis positive.

\subsubsection{Evaluation of RBV Curative Effect}

Cure: (1) The clinical symptoms completely disappeared. (2) The amine odor test was negative. (3) The $\mathrm{pH}$ of the secretion is $<4.5$. (4) Secretory clues are negative. Effective: The clinical symptoms are relieved and the secretion clues are negative or suspicious. Invalid: No significant reduction or even aggravation of clinical symptoms and signs. Microscopic examination of clue cells $(+)$.

\subsection{Treatment}

\subsubsection{Pelvic Floor Rehabilitation [6-7]}

Give pelvic floor muscle electrical stimulation + biofeedback and guide Kegel exercise for a long time. The use of low-frequency $\mathrm{AC}$ can be modulated, the waveform is a Bi-directional square wave. The instrument used is the USB4 type in the French PHENIX neuromuscular stimulation device series. Type I muscle stimulation frequency $8 \sim 33 \mathrm{~Hz}$, pulse width $320 \sim 740$ us, $\mathrm{R}$ (rest time) $=\mathrm{T}$ (working time); type II muscle stimulation frequency $20 \sim 50 \mathrm{~Hz}$, pulse width $160 \sim$ 
320 us, $\mathrm{R}=2 \mathrm{~T}$. First of all, low-frequency electric stimulation was given. The duration of type I muscle stimulation was 20 min, type II muscle electrical stimulation was $10 \mathrm{~min}, 2$ times/week, 5 times in total; the 6th time began to receive electrical stimulation + biofeedback, 5 times firstly. Myoelectric stimulation for $10 \mathrm{~min}+$ biofeedback for $20 \mathrm{~min}$, followed by 5 types of muscle electrical stimulation for $10 \mathrm{~min}$ + biofeedback $20 \mathrm{~min}, 2$ times/week, 10 times. The total treatment of 15 times. From the 6th time, Kegel was instructed to exercise. According to the individual's physical condition, the levator ani was recommended to be 150 to 200 times per day, once per day or distribution times. The evaluation of pelvic organ prolapse was taken according to the pelvic organ prolapsed quantitative staging system (POP-Q) developed by the American College of Obstetrics and Gynecology.

\subsubsection{Control Group Is Drug Treatment Group}

(i) RVVC drug treatment plan [1]

(1) Miconazole suppository $200 \mathrm{mg}$ is placed deep in the vagina, 1 capsule/night, for 7 to 14 days. Oral fluconazole 150 $\mathrm{mg}$ is given for the first time and served once every 72 hours. (2) Maintenance therapy: clotrimazole suppository $500 \mathrm{mg}$, once a week for 6 months; fluconazole $150 \mathrm{mg}$ once a week for 6 months; itraconazole $400 \mathrm{mg}$ once/month or $100 \mathrm{mg}$ once/week, for a total of 6 months; one of the maintenance plan may be selected.

(ii) RBV drug treatment program [1]

(1) Oral regimen: metronidazole $400 \mathrm{mg}$, twice a day for 7 days; alternative medicine available tinidazole $2 \mathrm{~g}$ once a day for 3 days; or tinidazole $1 \mathrm{~g}$ once a day, even for 5 days; or clindamycin $300 \mathrm{mg}$ twice a day for 7 days.

(2) Vaginal drug program: metronidazole suppository 200 $\mathrm{mg}$, once at night, or $2 \%$ clindamycin ointment rubbed, $5 \mathrm{~g} /$ time, a total of $7 \mathrm{~d}$.

1.5 statistical methods use SPSS 16.0 software package for statistical analysis, use chi-square test and t test, $\mathrm{P}<0.05$ for the difference that was statistically significant.

\section{Results}

\subsection{The Improvement of Pelvic Floor Muscle Strength of Pelvic Floor Rehabilitation Group Before and After Treatment}

Before the treatment, type I muscle strength in grades 4 and 5 in 4 cases, type II muscle strength in grades 4 and 5 in 8 cases; after treatment, type I muscle strength in grades 4 and 5 were 59 cases, and type II muscle strength in grades 4 and 5 in 60 cases $(\mathrm{P}<0.001)$. See Table 1 .

Table 1. Changes in pelvic floor muscle force before and after treatment in pelvic floor rehabilitation group (case),

\begin{tabular}{lllllllllllll}
\hline \multirow{2}{*}{ Group } & \multicolumn{1}{l}{ Muscle fiber I } & \multicolumn{1}{c}{ Muscle fiber II } \\
\cline { 2 - 13 } & $\mathbf{0}$ & $\mathbf{1}$ & $\mathbf{2}$ & $\mathbf{3}$ & $\mathbf{4}$ & $\mathbf{5}$ & $\mathbf{0}$ & $\mathbf{1}$ & $\mathbf{2}$ & $\mathbf{3}$ & $\mathbf{4}$ & $\mathbf{5}$ \\
\hline Before & 1 & 41 & 8 & 7 & 4 & 0 & 1 & 33 & 14 & 5 & 4 & 4 \\
After & 0 & 0 & 0 & 2 & 27 & 32 & 0 & 0 & 0 & 1 & 19 & 41 \\
Chi-squre & 100.99 & & & & & 90.87 & & & & \\
P value & $<0.001$ & & & \multicolumn{1}{c}{$<0.001$} & & & & \\
\hline
\end{tabular}

\subsection{Changes in Pelvic Organ Prolapse Before and After Treatment in the Pelvic Floor Rehabilitation Group}

Anterior prolapse of the vagina before and after treatment was 53 cases and 4 cases respectively $(\mathrm{P}>0.05)$. The difference was not statistically significant; the prolapse of the posterior vaginal wall was 25 Cases and 11 cases $(\mathrm{P}<0.001)$; uterine prolapse before and after treatment were 14 cases and 2 cases $(\mathrm{P}<0.001)$, the difference was statistically significant.

\subsection{The Recurrence of Vaginitis After Treatment}

After the use of pelvic floor rehabilitation techniques and follow-up observation for 1 year, only 2 patients with RVVC relapsed once or twice, but the clinical symptoms were significantly reduced with a recurrence rate of $3.3 \%$. Fifteen of 60 patients in the drug treatment group had recurrent episodes during normative drug use and one year afterwards. The frequency of relapses ranged from 4 to 8 times, with an average annual incidence of 5.35 times, and the relapse rate after drug treatment was $25 \%$. The difference between the two groups was statistically significant $(\mathrm{P}<0.05)$. In addition, liver damage occurred in 4 patients with RVVC in the drug treatment group, with an incidence of $5.12 \%$.

\section{Discussion}

The bactericidal effect of imidazole antifungal drugs widely used clinically is mainly against candidiasis albicans, and the bactericidal effect against candidiasis glabrata is weak [8-9]. The minimum inhibitory concentration (MIC) of candidiasis has significant interspecies differences. The MIC of imidazole antifungal drugs against candidiasis glabrata is 2 to 4 times that of candidiasis albicans; the majority of clinical diagnosis depends on microscopic examination and lack of fungal culture. As well as the identification of strains, treatment of infections caused by various candidiasis species often uses the same drugs, doses, and duration of treatment, often affecting the treatment of non-Albicans infections. Inadequate drug treatment leads to changes in the MIC of candidiasis to the drug, so that the drug-binding target enzyme on the cell membrane of candidiasis changes happened. Chong et al [9] found in the study that the gene mutations occurred in the recurrent infections of the strains, resulting in a decrease in the rate of imidazole and their combination, which led to an increase in the proportion of non-albicans to Candida [10]. Therefore, inappropriate drug treatment does not restore and utilize the self-purification and reconstitution capacity of the vagina, which may be one of the factors of RVVC. The bacteria that cause RBV are mainly vaginal inflammation caused by the imbalance of vaginal endophyte flora caused by excessive proliferation of anaerobes such as Gardneria, Corynebacterium parvum, etc., and are mixed infections caused by dysregulation of the normal flora in the vagina. When vaginal mucous membrane damage, various causes of vaginal $\mathrm{pH}$ increase is not conducive to growth of lactobacilli, Gardnerella and other anaerobic bacteria multiply, therefore, $\mathrm{BV}$ infection and vaginal defense machine, the damage caused 
by the system [1], if it repeatedly causes the factors that cause the destruction of the vaginal environment, it will become RBV infection. Drug therapy can suppress excessively proliferating anaerobic flora to rebalance the vaginal flora, but it cannot correct the cause of the destruction of the vaginal defense mechanism. According to the Cochrane Review, female pelvic floor muscle exercises should be performed for at least 3 months, and the pelvic floor conditions will be greatly improved [11-12].

As a result, Kegel's workout recommended continuous adherence for at least 3 consecutive months. The common basis for the pathogenesis of RVVC and RBV is the imbalance of vaginal flora. This study uses pelvic floor rehabilitation techniques such as low-frequency electrical stimulation, biofeedback, and Kegel exercises to help postpartum women recover normal vaginal structures, increase their ability to self-control pelvic floor muscles, and enhance the resistance to vaginal mucosa. The use of pelvic floor rehabilitation methods for RVVC and RBV patients in the non-acute phase of pelvic floor rehabilitation therapy, and observe the recurrence rate of vaginal inflammation after treatment, this study uses low-frequency electrical stimulation, biofeedback and Kegel exercise, through the RVVC Patients with RBV were treated with pelvic floor rehabilitation in non-acute phase of inflammation, and the recurrence rate of vaginal inflammation after treatment was followed up for 1 year. The results showed that the rehabilitation treatment significantly increased the pelvic floor muscle strength, and the pelvic organs, especially the vaginal wall and urethra, were restored. The vagina and vaginal structures that had been loosened due to pregnancy and childbirth were restored and the vaginal barrier function was restored. The patient's ability to self-control pelvic floor muscles enhances the resistance of the vaginal mucosa. At the same time follow-up and comparison of inflammatory recurrence of patients in the pelvic floor rehabilitation group and the control group at 1 year after treatment, the recurrence rates were respectively $3.3 \%$ and $25 \%$, and the pelvic floor rehabilitation group was significantly lower than the control group, indicating that physical therapy combined with drug treatment can prevent vaginal bleeding. The recurrence of inflammation is better than simple drug treatment. The results of this study are quite satisfactory.

\section{Conclusion}

For patients with vaginitis with recurrent episodes of endometabacterial dysbacteriosis, improvement of pelvic tissue structure, restoration of pelvic floor muscle strength and tension, repair of damaged mucosa, improvement of local disease resistance for at least 1 year or even longer Can effectively reduce the recurrence of this type of vaginal inflammation, it can be speculated that the use of appropriate pelvic floor rehabilitation therapy can reduce the use of antibacterial drugs, to avoid drug toxicity, reduce drug-resistant strains. At present, fewer cases are collected, and the follow-up time may not be sufficient, but at present the study can at least suggest that pelvic floor rehabilitation technology has positive significance in preventing and reducing the recurrence of RVVC and RBV.

\section{References}

[1] Xie Xing. Obstetrics and Gynecology [M]. 8th Edition. Beijing; People's Medical Publishing House, 2013: 248-250.

[2] Sobel JD. Vulvovaginal candidosis [J]. Lancet, 2007, 369(9577):1961-1971.

[3] Achkar JM, Fries BC. Candida infections of the genitourinarytract [J]. Clin Microbiol Rev, 2010, 23(2): 253-273.

[4] Ono F, Yasumoto S. Genital candidiasis [J]. Nippon Rinsho, 2009, 67(1): 157-161.

[5] El Din SS. Reynolds MT, Ashbee HR. et al. An investigation in -to the pathogenesis of vulvovaginal candidosis [J]. Sex TransmInfect, 2001, 77(3): 179-183.

[6] Su Yuanyuan, Han Yanhua, Li Danyan. Female pelvic floor function and evaluation method of pelvic floor muscle function [J]. Chinese Journal of Practical Gynecology and Obstetrics, 2015, 31(4):310-313.

[7] Zhang Jie, Wang Zhongmin, Ma Le, et al. Stress urinary incontinence caused by imbalance of levator ani muscle tension on both sides of postpartum [J]. Chinese Journal of Practical Gynecology and Obstetrics, 2015, 31(12): 1172-1173.

[8] Ferreira C, Silva S, Faria-Oliveira F, et al. Candida albicansvirulence and drug- resistance requires the 0 acyltransferase Guplp [J]. BMC Microbiol, 2010, 10: 238-240.

[9] Chong PP, Lee YL, Tan BC, et a1. Genetic relatedness of Can-dida strains isolated from women, IVith vaginal candidiasis in Malaysia [J]. J Med Microbiol, 2003, 52(Pt 8):657-666.

[10] Lockhart SR, Rccd BD, Pierson CL, et al. Most frequent scenario for recurrent Candida vaginitis is strain maintenance with "substrain shuffling": demonstration by sequential DNA finger- printing with probes Ca3, C 1, and CARE2 [J]. J Clin Micro-biol, 1996, 34(4):767-777.

[11] Li Ping. The risk of bacterial vaginitis on premature delivery and miscarriage [J]. Foreign Medical Intelligence, 1994, 15(18):98-99.

[12] Hay-Smith EJ, Dumoulin C. Pelvic floor muscle training versus no treatment, or inactive control treatments, for urinary incontience in women [J]. Cochrane Database Syst Rev, 2006, 25: CD005654. 